\title{
Methods of spectral density estimation for airfield pavements
}

\author{
Van-Hieu Nguyen ${ }^{1, *}$, Duy-Dong Nguyen ${ }^{1}$ and Vladimir Tatarinov ${ }^{2}$ \\ ${ }^{1}$ Le Quy Don Technical University, 236 Hoang Quoc Viet St., Ha Noi, Viet Nam \\ ${ }^{2}$ Moscow automobile and road construction state technical university (MADI), 64, Leningradsky \\ prospect, Moscow, Russia
}

\begin{abstract}
The paper gives an analysis of methods for estimating the spectral density of airfield pavements. The spectral density of the surface of the airfield pavement is the main criterion for estimating the roughness. This characteristic is used for the dynamic strength calculation of pavement. The paper presents analysis and comparison of the most popular methods for estimating the spectral density, and considers Burg's perspective parametric method, previously not used in aerodrome design practice.
\end{abstract}

\section{Introduction}

The most important element of the modern civil aviation airport is the airfield, designed to perform take-off and landing operations. In the design and operation of civil airports, the main task is the calculation of the strength of airfield pavements and assessing their operational status $[10,11,12,13,16]$. The solution of these problems requires an assessment of the unevenness of the surface of the aerodrome. When calculating the strength of airfield pavements, this assessment is necessary to determine the dynamic load from the wheel of the aircraft. The main cause of the dynamic forces are irregularities in the surface of the airfield. For runways and airfields of airports open to international flights, a generic feature of flatness of the airfield pavements $R$, the roughness index, must be defined $[10,12,17]$. Pavements of aerodromes are not allowed to be used, if the roughness index $R$ is less than 2. Check of the evenness of the runways is carried out at the newly built airfields and existing airfields after reconstruction or major repairs.

The roughness index depends on the characteristics of the spectral density estimation ( $C$ - level; $k$-slope); however, the method for determining these characteristics has not been specified in the normative documents $[10,12,17]$. They noted only that these coefficients should be obtained as a result of the spectral analysis of a number of marks obtained during geodetic measurements:

$$
S(\omega)=\frac{C}{\omega^{k}},
$$

where $S(\omega)$ - spectral density estimation; $\omega$ - spatial frequency - the reciprocal of the wavelength of roughness.

\footnotetext{
*Corresponding author: hieucse@ mail.ru
} 
No conditions on $k$ are imposed. However, the classical definition of spectral density consists in the fact that this is a real and even function; its representation is in the form [14]. Therefore this expression is only admissible for values of $k$ equal to even numbers $(2,4$ ...); the fractional value of $k$ will result in $S(\omega)$ not being an even function, and consequently, a spectral density.

Thus for almost half a century, it has been conventional to characterize the roughness of airfield pavements by the spectral density of the section of aerodrome element. Despite such a long period, this problem remains very relevant, because there is no unified method for estimating the spectral density.

The absence of clear and unambiguous methods for estimating the spectral density leads to the fact that the results obtained by different organizations when processing the same data can vary significantly. Thus, to solve the problem of statistical estimation of the spectral density of the aerodrome surface, it is necessary not only to perform analysis of the most popular methods, but also to choose the most stable and correct approach.

\section{Methods}

In modern practice of statistical processing of random processes, two main groups of methods are used: parametric and nonparametric.

Among the parametric methods, the most widespread are the following:

The classical method - The method consists in the preliminary determination of the correlated function and the subsequent estimation of the spectral density using Fourier transform. The classical method was used as the basis for almost all pioneering works on the investigation of the spectral composition of the unevenness of the surface of airfield and road pavements [15]. Despite the apparent simplicity and convenience, the classical parametric method for estimating the spectral density, inherent in the first studies, is practically not used at present. The reason for this is, above all, the distortions of the initial implementation, which are introduced to its transformations during filtration and approximation; in addition, the problem of the complexity of the choice of the approximating expression for estimating the correlated function and the calculation of the approximation error leads to a very limited field of application.

Parametric estimation by the method of Burg [3, 4], proposed by the well-known statistician J.P. Burg in 1967. Burg's algorithm is one of the very first and most well-known algorithms of autoregressive spectral estimation. In the literature, this algorithm was called the "Maximum Entropy Algorithm", but it should be regarded as one of independent directions, based on the maximum entropy principle of Burg. Burg's algorithm is identical to the geometric algorithm and differs from it only in that it uses a different type of estimation of the reflection coefficient, namely, an estimate determined by the method of least squares. This estimate of the reflection coefficient is the harmonic mean of the coefficients of the partial correlation of the prediction errors forward and backward. It is shown in [3] that its modulus does not exceed unity, and this guarantees the production of a stable filter having only poles. Despite the fact that Burg's algorithm has been successfully used in various studies, the author has not succeeded in validating the application of this algorithm for solving the problem of estimating the spectral density of the pavements of highways or aerodromes. The analysis of this algorithm showed its following positive features: high resolution in the analysis of short signals, guaranteed stability of the calculated shaping filter and minimization of prediction error forward and backward. All this makes the Burg method very promising for solving the problem of estimating the spectral density of aerodrome pavements. In addition, the use of this method does not 
require the approximation of spectral density estimation points, and, therefore, reduces the distortions of the estimates obtained. The disadvantages of Burg's method are manifested, mainly, in the study of realizations of signals with periodic components.

The methods of nonparametric estimation of spectral density include:

Method of periodograms. This method was first proposed by A. Schuster [9] in 1889 to search for periodicity in records of meteorological observations, records of magnetic declination and a number of sunspot numbers. The following observation is based on the periodogram method: for the realization of a random process $q(l)$ known on the interval $[0, L]$, the spectral density is the average value of the square of the modulus of the amplitude of the expansion of the random function in a Fourier series:

$$
I(\omega)=\frac{1}{L}\left|\int_{0}^{L} e^{-i \omega l} \cdot q(l) \cdot d l\right|^{2} .
$$

The periodogram method, based on the direct application of the Fourier transform, does not require the evaluation of the correlation function and its subsequent approximation. However, this method has a significant drawback that does not allow us to apply it as an estimate of the spectral density. The periodogram estimation is effective (asymptotically unbiased), that is, with an increase in the interval $[O, L]$, the estimation of the mathematical expectation tends to the true value (3). The disadvantage is the inadequacy of the estimate (2), because the variance estimate does not tend to zero (4):

$$
\begin{aligned}
& \lim _{L \rightarrow \infty} M[I(\omega)]=\int_{-\infty}^{\infty} e^{-i \omega \tau} q(l) d l=S(\omega) ; \\
& \lim _{L \rightarrow \infty} D[I(\omega)]=S^{2}(\omega) .
\end{aligned}
$$

The inadequacy of the estimate (2) leads to its considerable fluctuation, and with increasing the interval $[O, L]$, this fluctuation manifests itself more and more.

The inadequacy of the estimate (2), as well as the need to perform a very large number of calculations to obtain it, led to the fact that the periodogram method was almost forgotten and was not used for a long time.

With the advent of high-speed digital computers and the implementation of the Fast Fourier Transform [5, 6, 7, 8] in the mid-1960s, several methods were developed to overcome the inconsistency of the estimate (2). These methods include, above all, the methods of Bartlett and Welch.

The modified Bartlett periodogram algorithm was developed by M.S. Bartlett $[1,2]$ as early as 1948. The algorithm is based on the following: if we split the implementation length $[O, L]$ into $n$ intervals of length $L_{0}$ and apply (2) for each of these intervals, then we obtain $n$ estimates of the spectral density. General theoretical recommendations for the choice of values $n$ and $L_{0}$ cannot be obtained, since the optimal value $L_{0}$ depends not only on the type of the desired spectral density, but also on the specific problem that is being solved.

Welch's method of modified periodograms. This algorithm was proposed by P.D. Welch [8]; its main difference from the Bartlett method is that the division of implementation into segments is carried out with overlapping in the range from $50 \%$ to $65 \%$, which allows to significantly increase the ensemble size of the sections for subsequent averaging. Welch not only modified the basic scheme of the Bartlett segmentation and averaging method by using 
overlapping segments, but also implemented the application of the data window. Before calculating the periodogram of each segment, this segment is processed using a data window. The purpose of the application of the window is to reduce the effects due to side lobes due to a slight deterioration in the resolution and to reduce the shift in the estimates. The purpose of overlapping segments is to increase the number of averaged segments for a given length of data recording, and thereby reduce the dispersion of the spectral density estimate. Based on the fast Fourier transform P.D. Welch also developed an efficient computational procedure for realizing his method of averaging the periodogram of weighted and overlapping data segments, and this made the Welch method the most popular periodogram method of spectral estimation today.

In the practice of estimating the spectral density of aerodrome pavement, the Welch method is used most often, but the results of the estimation by this method need to be approximated by an analytical expression, for example, (1). The approximation process introduces subjective distortions into the results obtained due to the uncertainty of the method, the number of points, and also the weight of each point. This leads to the fact that the results of processing the same source data may differ significantly.

In the Burg algorithm, almost all of the drawbacks inherent in the Welch method are absent, since a parametric estimate based on the best model that does not require approximation is performed and immediately leads to an estimate of the spectral density of the form:

$$
S(\omega)=\frac{C}{\omega^{2}} .
$$

This paper presents a study of the estimate of the spectral density of the airfield pavements of one of the airports in Vietnam ("Cam Ranh" airport), obtained by various methods. To obtain the initial data about the surface of the airfield pavement, a method of complex geometric leveling of the runway in the longitudinal direction was adopted. The leveling was carried out in 2015 [14]. The leveling step, taken from the smoothing ability of the wheel pneumatics of the main support of the aircraft, was taken equal to $0.5 \mathrm{~m}$ (Figure $1)$.

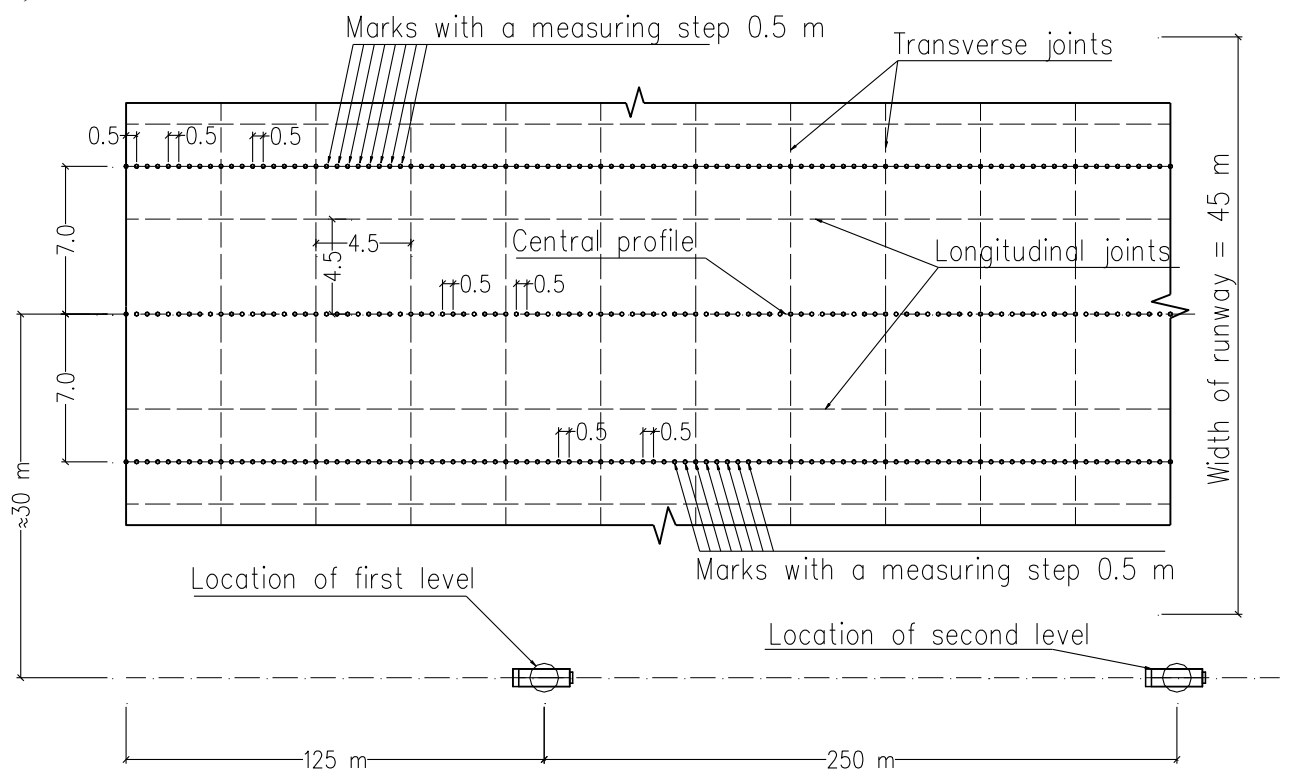

Fig. 1. Geometric leveling of the runway of airport "Cam Ranh". 
The longitudinal profiles obtained as a result of leveling are shown in Fig. 2.

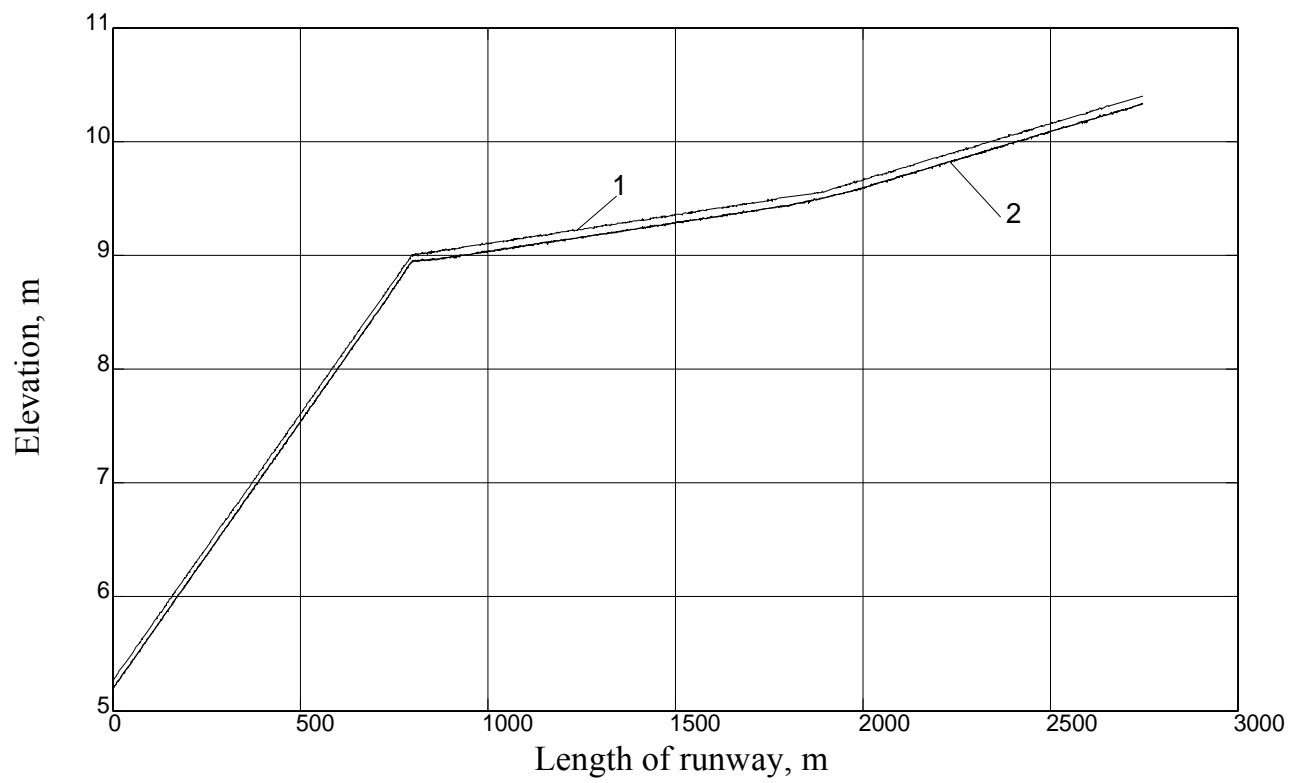

Fig. 2. Results of geometric leveling of the airport "Cam Ranh" runway;

1 - longitudinal profile along the axis of symmetry of the runway; 2 - longitudinal profiles along $7 \mathrm{~m}$ sections.

A package of computer programs in the "MATLAB" system was created to carry out a statistical estimation of the spectral density of roughness for airfield pavements.

\section{Results and Discussion}

The obtained data of geometric leveling of the runway surface of the "Cam Ranh" airport were used to estimate the spectral density by the previously considered methods: periodogram, Welch and Burg. The purpose of these studies is not only to obtain an estimate of the spectral density needed to determine the leveling index, but also to compare the results obtained by different algorithms, taking into account their features, merits and demerits.

When estimating the spectral density by the periodogram method, its following features appear: this estimate is asymptotically unbiased, but it is not consistent. That is, the accuracy of determining the spectral density does not increase with the length of the realization, therefore, estimate (2) cannot be accepted as an estimate of the spectral density.

In Fig. 3 is a graph of the spectral density estimate by the periodogram method. The irregularity (fluctuation) of the evaluation also appears due to its inconsistency, and with increasing $L$ the irregularity becomes more and more pronounced. The inconsistency of estimating the spectral density is due to the fact that it is not a numerical parameter characterizing $S(\omega)$ that is estimated, but the entire course of this function is estimated, that is, a nonparametric estimate of $S(\omega)$ is made.

We also estimated the spectral density using the most popular nonparametric Welch algorithm and the Burg parametric algorithm, which is well known, but was used for the first time to estimate the spectral density of the pavement of the aerodrome runway. 


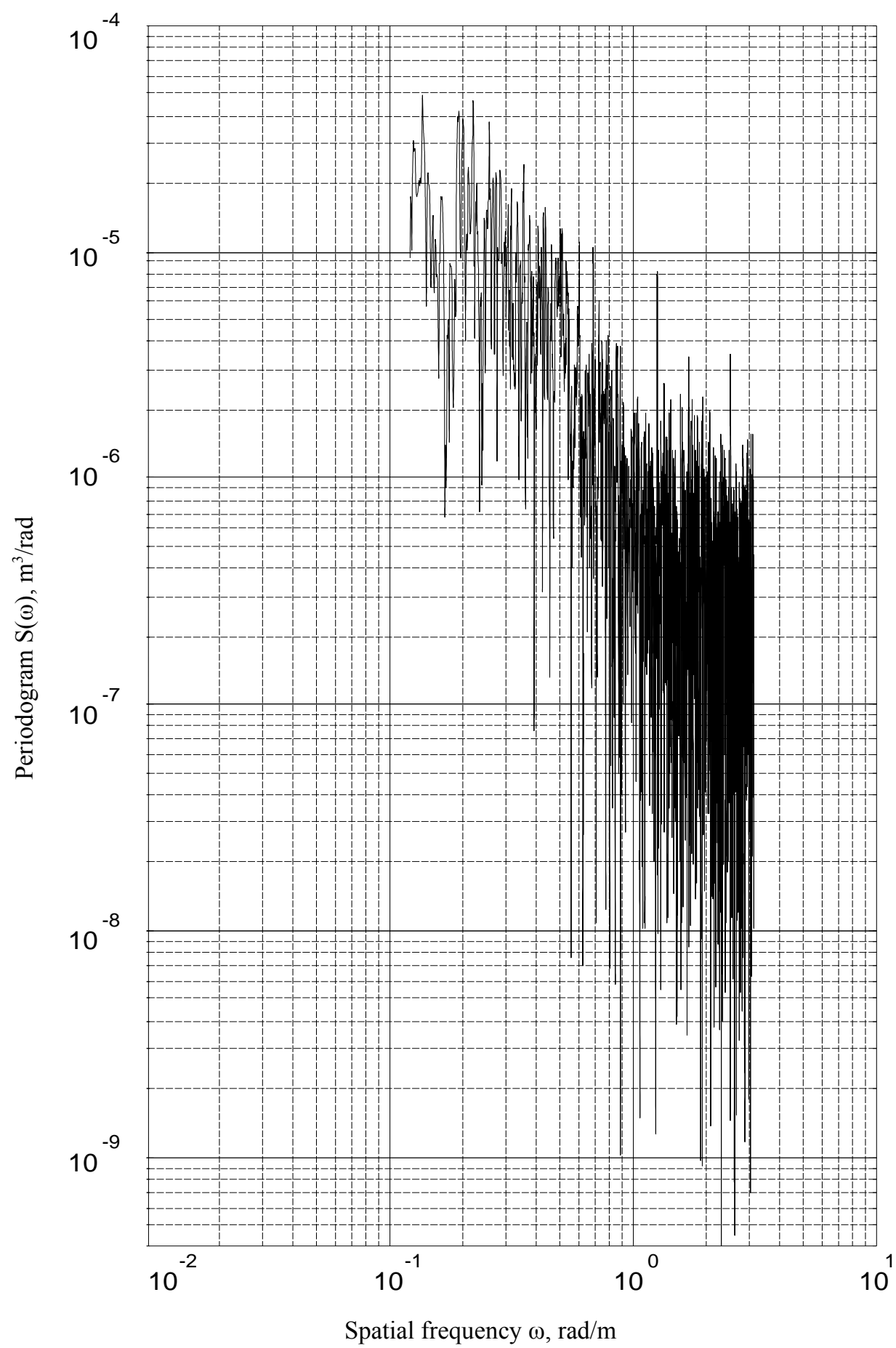

Fig. 3. Estimation of spectral density by the periodogram method.

Subsequently, estimates of the spectral density by the methods of Welch and Burg were obtained, while the Welch's estimates were approximated by the expression (5). The graphs of the estimates are shown in Fig. 4. 


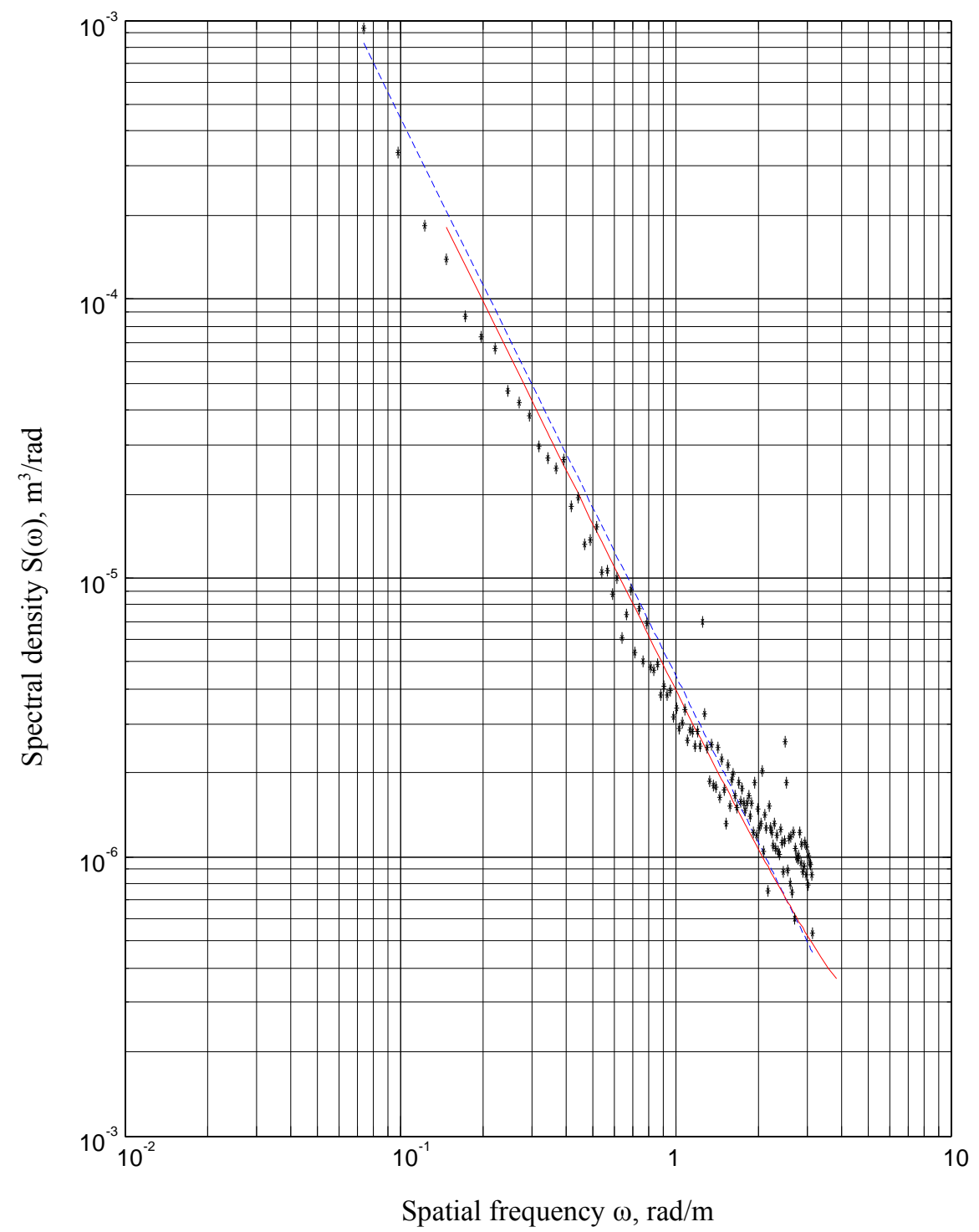

Fig. 4. Estimation of spectral density by the method of Whelch and Burg;

***Welch's assessment; --- approximation of the Welch's estimation; --- Burg's estimation.

Calculations using the Welch algorithm were performed under the following assumptions: the number of segment points is 256 , the overlap of segments is $50 \%$, the spectral window - the Hann window, the method of approximation - the method of least squares. It should be noted that with other parameters (assumptions) in calculation, the spectral density estimate will be shifted relative to the obtained value. When using the Burg algorithm, no assumptions are required.

Based on the analysis of the obtained estimates, obtained in this calculation and in [14], the good convergence of the results obtained by the algorithms of Burg and Welch can be confirmed. At the same time, it should be noted that the Welch algorithm, in contrast to the Burg algorithm, requires approximation (averaging) of the results obtained. The approximation procedure must be performed for the same number of points in all frequency 
ranges; otherwise, the estimate may shift. In addition, when applying the Welch algorithm, it is necessary to determine in advance not only the number of intervals to which the entire original array will be divided, but also the overlapping area of the indicated intervals.

As a result of the performed studies and calculations, the level of spectral density determined by the Burg algorithm was $4.0 \cdot 10^{-6} \mathrm{~m} \cdot \mathrm{rad}$, and according to the Welch algorithm with the assumptions made $-4.1 \cdot 10^{-6} \mathrm{~m} \cdot \mathrm{rad}$, but it should be noted that the Burg algorithm is not only more stable than the Welch algorithm, since it does not require additional calculation conditions, but also is realized when performing significantly fewer computational operations. The leveling index determined according to the methods $[10,12]$ was 4.62, which indicates the suitability of the "Cam Ranh" airport pavements for operation.

\section{Conclusions}

1. It has been conventional to characterize the roughness of airfield pavements by the spectral density of the section of aerodrome element;

2. The estimation of spectral density is used not only as a characteristic of roughness of airfield pavements, but also as the basic function for calculating of dynamic loads from the wheels of the airplane on airfield pavements;

3. The use of expression (1) in the estimation of the leveling index is unreasonable, since for a fractional value of $k$ expression (1) ceases to be an even function, and, consequently, can not be an estimate of the spectral density. In this case, we should use formula (5);

4. The Welch algorithm, in contrast to the Burg algorithm, requires approximation (averaging) of the results obtained. In addition, when applying the Welch algorithm, it is necessary to adopt a series of assumptions that must be the same for any calculation; the application of the Burg algorithm is free of drawbacks, leading to an inconsistency and a shift in the estimation of the spectral density;

5. Thus, we can conclude that the Burg algorithm is the most optimal for estimating the spectral density of the pavement of the aerodrome under study. The application of the Burg algorithm in the design and operation of civil aerodromes avoids the errors caused by the uncertainty inherent in other methods.

\section{References}

1. M.S. Bartlett, Periodogram Analysis and Continuous Spectra. Biometrika. Vol. 37. Pp. 1-16. (1950)

2. M.S. Bartlett, Smoothing Periodograms from Time-Series with Continuous Spectra. Nature. Vol. 161. Pp. 686-687. (1948)

3. J.P. Burg, A New Analysis Technique for Time Series Data. NATO Advanced Study Institute on Signal Processing with Emphasis on Underwater Acoustics, Aug. (1968)

4. J.P. Burg, Maximum Entropy Spectral Analysis, paper presented at the 37th Annual International Meeting, Soc. of Explor. Geophysics, Oklahoma City, Oct. (1967)

5. C. Bingham, M.D. Godfrey, J.W. Tukey, Modern Techniques of Power Spectrum Estimation. IEEE Transactions on Audio and Electroacoustics. Vol. AU-15. No 2. Pp. 56-66. (1967)

6. J.W. Cooley, J.W. Tukey, An Algorithm for the Machine Calculation of Complex Fourier Series. Mathematics of Computation. Vol. 19. No. 90. Pp. 297-301. (1965) 
7. J.W. Cooley, P.A.W Lewis, P.D. Welch, The Fast Fourier Transform and Its Applications. IEEE Transactions on Educations. Vol. 12, No 1, Pp. 27-34. (1969)

8. P.D. Welch, The Use of Fast Transform for the Estimation of Power Spectra: A Method Based on Time Averaging Over Short, Modified Periodograms. IEEE Transactions on Audio and Electroacoustics. Vol. AU-15. No.2. Pp. 70-73. (1967)

9. A. Schuster, On the investigation of hidden periodicities with application to a supposed 26 day period of meteorological phenomena. Terrestrial Magnetism and Atmospheric Electricity. Vol. 3. No. 1. Pp. 13-41. (1898)

10. Order of the Ministry of Transport of Russia dated August 25, 2015 No. 262. On approval of the federal aviation regulations "Requirements for aerodromes intended for take-off, landing, taxiing and parking of civil aircraft". 148 p. (2015)

11. Guide to the design of aerodromes. Part 3 Coatings. ICAO Doc. 9157 - AN / 901. Updated version (update date 01/01/2018), Montreal: ICAO. 349 p. (2018)

12. Guidelines for the operation of civil airfields of the Russian Federation (REGA RF-94). M .: Air transport, 231 p. (1995)

13. SP 121.13330.2012 Airfields. Updated edition of SNiP 32-03-96, Ministry of Regional Development of Russia. - M, 98 p. (2012)

14. Nguyen Van Hieu. Improving the method of dynamic calculation of rigid airfield pavements for SRV conditions. Diss. On the competition. scholarly step. Candidate of Technical Sciences: Spec .: 05.23.11. Moscow. 165 s. (2017)

15. Khachaturov A.A. Dynamics of the road - tire - car - driver system. / A.A. Khachaturov. M., mechanical engineering. 535 p. (1976)

16. TCVN 10907: 2015-Sân bay dân dụng-Mặt đường sân bay-Yêu cầu thiết kế. Hà Nội, (2015)

17. TCVN 8753: 2011-Sân bay dân dụng-Yêu cầu chung về thiết kế và khai thác. Hà Nội, (2011) 